\title{
Design and Implementation of the Networked measurement and Control System based on the Internet
}

\author{
Shengqian Ma Weibing Liu Xiaoyong Li \\ Key laboratory of Atomic and Molecular Physics \& Functional Materials of Gansu Province, College of Physics and \\ Electronic Engineering, Northwest Normal University \\ Lanzhou, 730070, China \\ e-mail: s.q.ma@163.com; iamliuweibing@163.com
}

\begin{abstract}
Distributed and networked measurement and control system has become a trend. In this paper, we designed and implemented an Internet-based networked measurement and control system. High-speed Ethernet plays the role of the field measurement and control subsystem. In this subsystem, we use the gateway node to manage the data acquisition and control units and through it to communicate with the Server on the Internet. The Internet Server, by providing the web service interface, can communicate with the third-party applications. We used C8051F340 as the core to design a control node, which achieved the coordinately call and execution efficiently based on event driven mechanism. In the software design aspect, the gateway node has joined the function of parameters configuration, which reduced the influence on the network bandwidth to the control system and improved the efficiency of measurement and control system. The web server was designed so that the gateway node can maintain the normal operation of the field measurement and control subsystem which only need the Internet server sends the configuration information to the corresponding gateway node. We verified the validity of this system through networked measurement and control of temperature and humidity.
\end{abstract}

Keywords- Measurement and Control Node; Event Driven; Gateway node; Web Server; Networked Control System

\section{INTRODUCTION}

The development trend of measurement and control system is intelligent, remote and networked. Measurement and control network transition from original centralized mode to be distributed mode, becoming a control system with open, interoperable, decentralized, networked and intelligent [1]. Researchers worldwide have established some distributed measurement and control applications. University of Barcelona has realized a CAN bus network capable application processor with a 16-bit microcontroller and a smart transducer interface module with an 8-bit microcontroller. Jadavpur University has provided a general scheme for development of network capable smart transducer interface in multi-drop field bus environment [2].

The measurement and control system based on network has been divided into field bus control system and the measurement and control system based on the Internet. Field bus control system use field bus to connect all the controller and instrument equipment, which is an open, with interoperability and completely scattered distributing measurement and control system. It is often used in the industrial producing or other real-time high-demanding fields[3,4]. In recent years, with the development of network communication technology, Ethernet enters the industrial control field, and plays a more and more important role, forming a new type of Ethernet control network technology[5].

The design of measurement and control system based on the Internet used high-speed Ethernet on the realization of field measurement and control subsystem. Subsystems interconnect with internet server through the network interconnection equipment formed a integrated control system with the functions of measurement, control and management [6]. This method is an important direction for the development of measurement and control system based on Internet in the future. In theory, it is able to attain all of the goal that modern control system can do as long as the Internet is stable enough and the communication bandwidth is large enough and becomes a control system that is not subject to regional restriction and meet all kinds of real-time demand. Its development prospects are very broad[7]. Network control based on the event-driven mode can reduce the influence of network bandwidth on the control system and improve the system efficiency, but the hardware implementation of the event driven is not easy[8,9].

In networked control application, most of these solutions have put emphasis on the hardware implementation of separate modules and lack high-level distributed applications in specific industry automation environments. The network control nodes designed in this work has not only the functions of measurement, control and display, but also has the similar structure and communication model with the information network. This paper describes a design of a network measurement and control system based on the Internet. In the software aspect, this paper designed an event driven mechanism suitable for the node, realized the efficient coordination and also call and execution between software modules. In the hardware aspect, we expanded the scope of application of the measurement and control node through reservation interface on the chip.

\section{THE DESIGN SCHEME OF THE CONTROL SYSTEM}

As is shown in Fig. 1, the whole system can be divided into three parts from topology structure: (1) field measurement and control system is composed by control node, (2) gateway nodes consists of control layer, (3) the information processing layer is composed by Internet server. Considering the control system from the function aspects: 


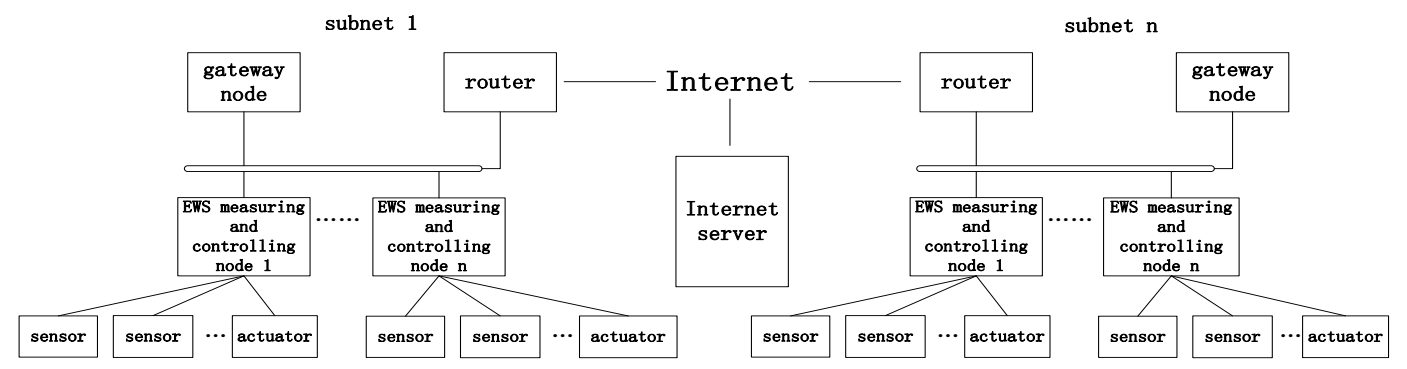

Figure 1. The topology structure of the system

control node is responsible for collecting sensor values and receives the control command from the control layer, realizing control operation to the controller; gateway nodes consist of field control layer of the control system, which is not only a part of field control subsystem, but also the communication media between control subsystem and the information processing layer. On the one hand, receiving configuration information from the Internet server to realize the automatic control of the field control subsystem, on the other hand they sent a variety of data (including the sensor data, the controller state information, parameter configuration information and etc.) about field control subsystem to the Internet server. The Internet server is located in the information processing layer of the measurement and control system. The managers can login to the Internet server and manage the operation of the whole system.

\section{HARDWARE DESIGN OF THE CONTROL NODE}

\section{A. Overall Design}

As is shown in Fig. 2, the hardware core is mainly composed of four parts, data acquisition and signal conditioning parts consist of the sensor and signal conditioning circuit, control center consist of C8051F340 MCU and its accompanying hardware, network communication parts consist of network controller CP2200 and its accompanying hardware, as well as the temperature and humidity control parts.

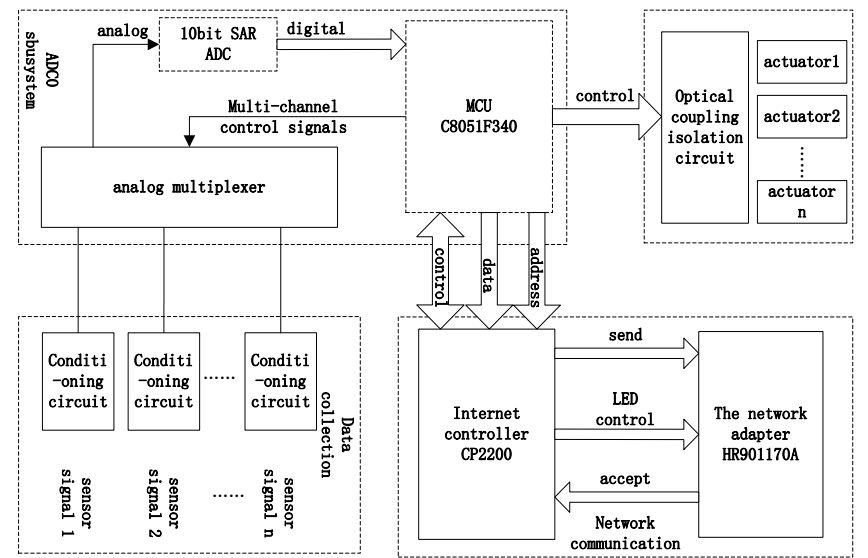

Figure 2. The diagram of measurement and control node

\section{B. Interface Design of C8051F340 and CP2200}

C8051F340 and CP2200 interface as shown in Fig. 3. In order to make CP2200 working in non multiplexed Intel bus format, CP2200 MOTEN and MUXEN pins are connected to the ground. The P3 and P4 port serve as 8 bit address and 8 bit data lines are connected to CP2200. If any one of the 14 interrupt events of the CP2200 is generated, CP2200 would set the corresponding interrupt flag of the interrupt status register to 1 and notify the host by driving /INT pin to a low level. The /INT pin will remain low level before all the interrupt flags of the allowed interrupts are cleared to 0 by host computer. The P3 and P4 port of C8051F340 MCU are respectively connected to address, data bus of the CP2200 network controller, and the P2.7 port serves as the chip selection; the P1.6 and P1.7 port serve as read and write control; the P0.2 port receives interruptions.

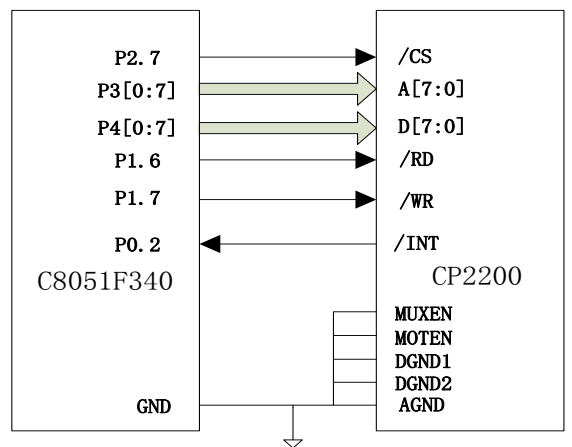

Figure 3. Interface between C8051F340 and CP2200

\section{Hardware Interface Design of CP2200 and RJ45 Network Adapter}

Special attention should be paid to the connecting circuit of CP2200 controller and RJ45, because these parameters have great significance on the reliability of communication. When connecting with RJ45, the pins are: RX+, RX-, TX+, TX-, and connecting to the network through RJ45. The LINK pin and ACT pin of CP2200 are respectively connected with LINK LED and ACT LED to indicate the state of the network, and LINK is effective when detecting a valid connection pulse, ACT is effective when transmitting or receiving a packet. 


\section{SOFTWARE DESIGN}

\section{A. Overall Design}

The software structure is mainly divided into measure and control node software structure, gateway node software structure and Internet server software structure.

\section{B. The Design of the Measure and Control Node}

The design idea of the software is based on event driven. In this program implementation, we first define an event type variable and many kinds of events constants, and then constantly judge whether incidents occurred in the event priority cycle. If there specific events occurred, do the corresponding operation and eliminate the effect brought by the corresponding event on the event type variables. Events generated from two sources: one is generating new events during the execution of the program, and the other is timer generating event initiatively. And finally the entire program is driven to run by this event generating mechanism.

\section{The Software Design of the Gateway Node}

The software is composed of the following modules: sensor network communication module, control network communication module, SQLite database operation module and system settings module of network communication. In order to improve the reuse rate of software code, the network communication function was singly designed as a module and the communication function was refined to control command. The communication between service program and measurement and control node, as well as the communication between service program and the Internet server was implemented through the combination of network communication and control command mode.

\section{The Software Structure of Internet Server}

The structure of Internet server is shown as Fig. 4. The software is composed of the modules: Http service request and response, Analysis and data processing, web service interface etc.

\section{E. The Design of Web Server}

In order to improve the visual intuition, the system needs a function that can render curves of the monitored temperature and humidity in real-time. We used Zed Graph

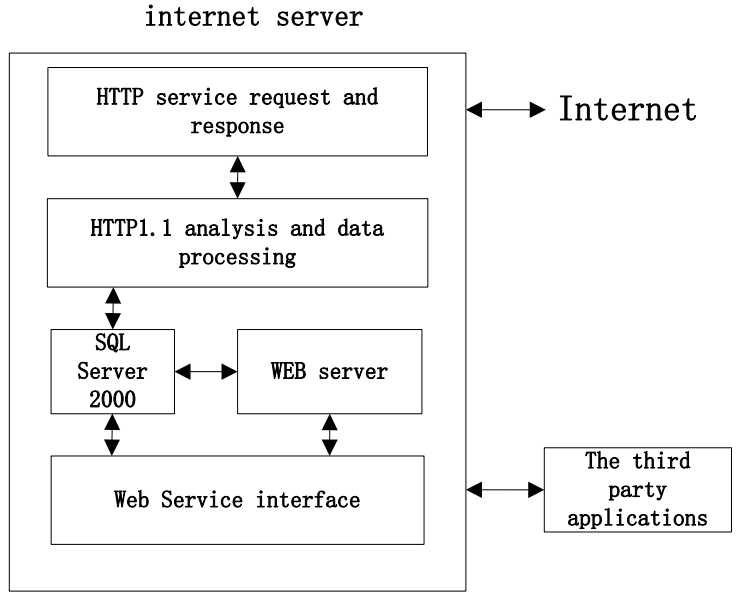

Figure 4. The structure of Internet server

charts based on .NET to achieve this function in this paper. In order to reduce the amount of the network communication data each time, improve the efficiency of data communication and enhance the user experience, it needs a suitable real-time web interactive technology based on the $\mathrm{B} / \mathrm{S}$ model. We chose Ajax technology, which is the most popular B/S interaction at present to achieve this function.

\section{SYSTEM TESTING}

In order to detect the performance of the system, we designed the temperature and humidity networked control system based on the Internet. The interface of the system on the Internet server includes the calendar, the current and historical curve, the sensor information list, the operating state indicator of current measure and control node, and the actuator information query and setting function. In Fig. 5 the Internet server gets the temperature values and draw the operation effect curve (similar to humidity interface). Two inquiry ways include the conditional query and the whole query. Query conditions consist of gateway node ID and measurement and control node ID. Fig. 6 is respectively corresponding to temperature and humidity controller setting. The execution state indicates that whether the actuator is currently in working state and the application of PID algorithm shows that whether the actuator used the PID

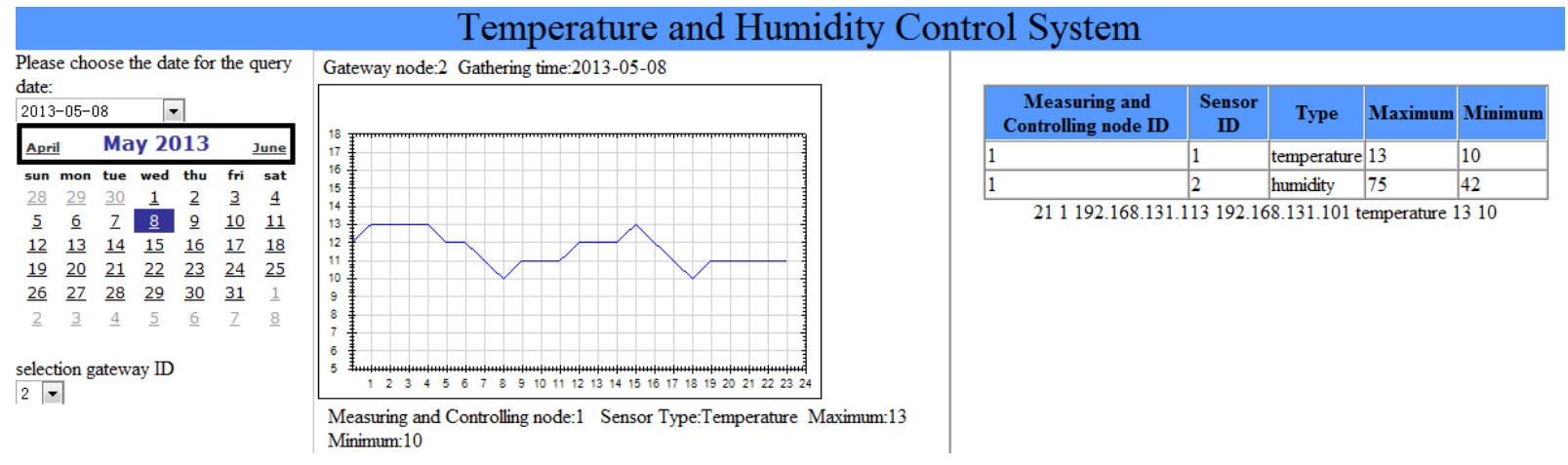

Figure 5. The control interface example of Internet server 
control algorithm. If the PID control algorithm is used, we need to set the PID parameter. In the case of default, the PID parameter settings are as follows: the proportion parameter is 0.5 , integral parameter is 1 , and differential parameter is 0 . The control target indicates the target value that PID control to achieve.

\begin{tabular}{|c|c|}
\hline Actuator details: & Actuator details: \\
\hline Actuator labeled: 1 & Actuator labeled: 1 \\
\hline Measuring and Controlling node ID: 1 & Measuring and Controlling node ID: 1 \\
\hline Measuring and Controlling node IP: 192.168 .131 .101 & Measuring and Controlling node IP: 192.168 .131 .101 \\
\hline Gateway node ID: 2 & Gateway node ID: 2 \\
\hline Gateway node IP: 192.168 .131 .101 & Gateway node IP: 192.168 .131 .101 \\
\hline Actuator Style: Temperature & Actuator Style: Humidity \\
\hline Executing state: & Executing state: \\
\hline Application of PLD algorithm: & Application of PLD algorithm: \\
\hline PLD parameter & PLD parameter \\
\hline Scale parameter: 0.5 & Scale parameter: 0.5 \\
\hline Integration Parameters: 1 & Integration Parameters: 1 \\
\hline differential parameter: 0 & differential parameter: 0 \\
\hline Target: 25 & Target: 70 \\
\hline
\end{tabular}

Figure 6. Setting temperature and humidity controller

The following are the results of using PID control. As shown in Fig. 7 is the change condition of the real-time temperature curve when temperature target value is set to 25 degrees Celsius, and we can see from the chart that the temperature is maintained at 25 degrees Celsius by heating device under the control of the PID. As shown in Fig. 8 is the change condition of the real-time humidity curve when relative humidity target value is set to 80 , and we can see the relative humidity maintained at 80 by humidification equipment under the control of the PID.

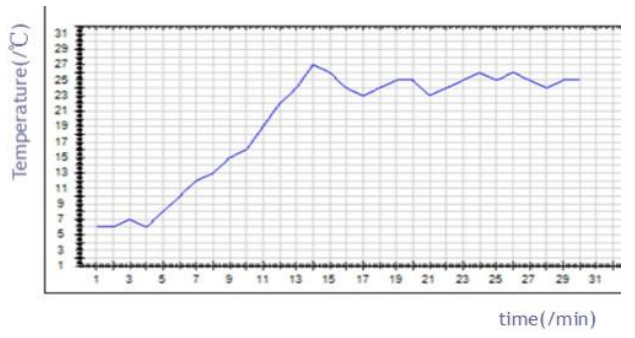

Figure 7. PID temperature control at 25 Celsius

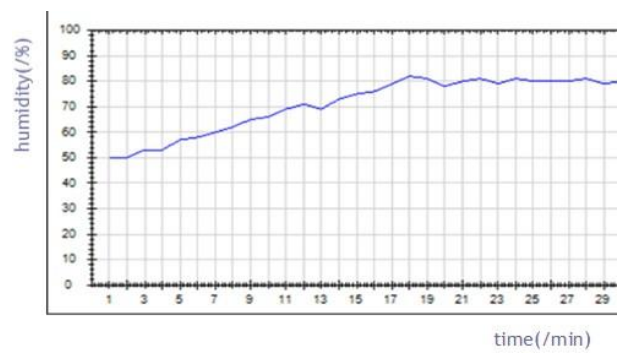

Figure 8 . PID humidity control relative humidity at 80

\section{CONCLUSION}

This paper describes the design and implementation of a network measurement and control system based on Internet. The hardware part mainly designed the measurement and control node of the measurement and control subsystem. And then, we designed the gateway node, which can not only realize data communication between field measurement and control subsystem and the Internet network, but also implement the control of the measurement and control subsystem. In the Internet server software system design aspect, we got the integration of measurement and control system with other software system by providing the Web Service measurement and control interface. Finally, we proved the validity of this system by comparing the actual results and the measurement and control system results. The design method of this system will have wide prospects in the networked measurement and control applications.

\section{ACKNOWLEDGMENT}

Author would like to acknowledge the support of the National Natural Science Foundation of China under grant NO. 61162017 and the Postgraduate Tutor Project of Universities of Gansu Province: NO. 1101-03.

\section{REFERENCES}

[1] Liu Zhigang, "Development and application of modern measurement and control technology," Mechanical and Electronic Information, iss.12, 2012, pp.120-121.

[2] S. Pal, A. Rakshit ,'Development of network capable smart transducer interface for traditional sensors and actuators," Sens. Actuators A:Physical, vol.112, iss.2-3, May.2004, pp.381-187.

[3] Y. Cetinceviz and R. Bayin dir, "Design and implementation of an Internet based effective controlling and monitoring system with wireless field bus communications technologies for process automation-An experimental study," ISA Transactions, vol.51, iss.3, May.2012, pp.461-470.

[4] R. Husemann and C. E. Pereira. "A multi-protocol real-time monitoring and validation system for distributed field bus-based automation applications," Control Engineering Practice, vol.15, iss.8, Aug.2007, pp.955-968.

[5] G.Cena, L.Seno, A.Valenzano and S.Vitturi, "Performance analysis of Ethernet Powerlink networks for distributed control and automation systems," Computer Standards \& Interfaces, vol.31, iss.3, Mar.2009, pp.566-572.

[6] S.Vitturi, "DP-Ethernet: the Profibus DP protocol implemented on Ethernet," Computer Communications, vol.26, iss.10, Jun.2003, pp.1095-1104.

[7] Xie Dong, Wen Yangdong and Bi Rui, "The design of embedded distributed remote control system based on Internet. Automation and instrumentation," Automation \& Instrumentation, iss.3, 2008, pp11-14.

[8] Hu Songlin and Dong Yue, "Event-triggered control design of linear networked systems with quantizations," ISA Transactions, vol.51, iss.1, Jan.2012, pp153-162.

[9] H.Yu and P.J.Antsaklis, "Event-triggered output feedback control for networked control systems using passivity: Achieving stability in the presence of communication delays and signal quantization," Automatica, vol.49, iss.1, Jan.2013, pp30-38. 